\title{
Abnormal Factor VIII Coagulant Antigen in Patients with Renal Dysfunction and in Those with Disseminated Intravascular Coagulation
}

\author{
Mark J. Weinstein, Leslie E. Chute, Gunther W. Schmitt, Robert H. Hamburger, \\ Kenneth A. Bauer, Joseph H. Troll, Paul Janson, and Daniel Deykin \\ William B. Castle Hematology Research Laboratory, Boston City Hospital, Boston University School of Medicine, Boston, Massachusetts \\ 02118, Boston Veterans Administration Medical Center, Boston, Massachusetts 02130, and the Beth Israel Hospital, Boston, \\ Massachusetts 02115
}

\begin{abstract}
Factor VIII antigen (VIII:CAg) exhibits molecular weight heterogeneity in normal plasma. We have compared the relative quantities of VIII:CAg forms present in normal individuals $(n=22)$ with VIII:CAg forms in renal dysfunction patients $(n=19)$ and in patients with disseminated intravascular coagulation (DIC; $n=7$ ). In normal plasma, the predominant VIII: CAg form, detectable by sodium dodecyl sulfate polyacrylamide gel electrophoresis, was of molecular weight $2.4 \times 10^{5}$, with minor forms ranging from $8 \times 10^{4}$ to $2.6 \times 10^{5} \mathrm{D}$. A high proportion of VIII:CAg in renal dysfunction patients, in contrast, was of $1 \times 10^{5} \mathrm{~mol}$ wt. The patients' high $1 \times 10^{5} \mathrm{~mol}$ wt VIII: CAg level correlated with increased concentrations of serum creatinine, $F_{1+2}$ (a polypeptide released upon prothrombin activation), and with von Willebrand factor. Despite the high proportion of the $1 \times 10^{5} \mathrm{~mol}$ wt VIII:CAg form, which suggests VIII:CAg proteolysis, the ratio of Factor VIII coagulant activity to total VIII:CAg concentration was normal in renal dysfunction patients. These results could be simulated in vitro by thrombin treatment of normal plasma, which yielded similar VIII:CAg gel patterns and Factor VIII coagulant activity to antigen ratios. DIC patients with high $F_{1+2}$ levels but no evidence of renal dysfunction had an VIII:CAg gel pattern distinct from renal dysfunction patients. DIC patients had elevated concentrations of both the $1 \times 10^{5}$ and $8 \times 10^{4} \mathrm{~mol}$ wt VIII:CAg forms. We conclude that an increase in a particular VIII:CAg form correlates with the severity of renal dysfunction. The antigen abnormality may be the result of VIII:CAg proteolysis by a thrombinlike enzyme and/or prolonged retention of proteolyzed VIII:CAg fragments.
\end{abstract}

\section{Introduction}

In the presence of Factor IXa, phospholipid, and calcium, Factor VIII (antihemophilic factor) accelerates the conversion of Factor $\mathrm{X}$ to Xa. Although diminished Factor VIII coagulant activity (VIII:C) $)^{1}$ has long been recognized as a major cause of inherited

Address correspondence to Dr. Weinstein, Boston City Hospital, Thorndike 508, 818 Harrison Ave., Boston, MA 02118.

Received for publication 10 April 1985.

1. Abbreviations used in this paper: DIC, disseminated intravascular coagulation; SDS-PAAGE, sodium dodecyl sulfate-polyacrylamide agarose gel electrophoresis; vWF, von Willebrand factor; VIII:C, Factor VIII coagulant activity; VIII:CAg, Factor VIII antigen.

J. Clin. Invest.

(c) The American Society for Clinical Investigation, Inc.

$0021-9738 / 85 / 10 / 1406 / 06 \quad \$ 1.00$

Volume 76, October 1985, 1406-1411 hemorrhagic disease, little is known about the association of Factor VIII structural abnormalities with disease processes. This relationship can now be examined by employing a recently developed $(1,2)$ immunological sodium dodecyl sulfate-polyacrylamide agarose gel electrophoresis (SDS-PAAGE) method to analyze Factor VIII antigen (VIII:CAg) molecular weight forms present in whole plasma.

Most VIII:CAg in normal plasma is noncovalently bound to von Willebrand factor (vWF), a large multimeric glycoprotein essential for proper platelet adhesion to subendothelium (3). VIII: CAg exhibits molecular weight heterogeneity in normal plasma: most VIII:CAg has an apparent molecular weight by SDSPAAGE of $2.4 \times 10^{5}$, with minor forms ranging in size from 8 $\times 10^{4}$ to $2.6 \times 10^{5} \mathrm{D}(2)$. VIII:CAg forms above $1 \times 10^{5} \mathrm{~mol}$ $w t$ are very sensitive to cleavage by a number of plasma enzymes including thrombin, Factor $\mathrm{Xa}$, protein $\mathrm{C}$, and plasmin. Depending on the enzyme, proteolytic cleavage of Factor VIII can both increase (4) and decrease (5) VIII:C coagulant activity. Thus analysis of changes in the quantity, structure, and activity of the highly labile protein could theoretically serve as a good indicator of in vivo serine protease generation.

We have surveyed a number of patient populations to determine if VIII:CAg abnormalities can be detected in disease states, if these abnormalities can be correlated with disease etiology, and if modification of protein form can be related to VIII: C. This report describes abnormal VIII:CAg molecular weight forms in patients with renal dysfunction and those with disseminated intravascular coagulation (DIC). The abnormal VIII:CAg form that occurs in renal dysfunction patients is distinct from that present in DIC patients and reflects the severity of renal disease.

\section{Methods}

Patients. Blood samples were drawn from four groups of individuals: 11 patients with varying degrees of renal dysfunction not requiring dialysis, 8 patients receiving regular hemodialysis, 7 DIC patients with normal renal function, and 22 normal individuals.

The average serum creatinine of renal patients not requiring dialysis was $6.0 \mathrm{mg} / \mathrm{dl}$ (range, $1.5-9.4 \mathrm{mg} / \mathrm{dl}$ ). Their ages ranged from 46 to 72 yr (mean, $58.7 \mathrm{yr}$ ). Four patients had hypertensive renal disease; three had diabetic nephropathy; and there was one patient with each of the following: focal sclerosis, lupus nephritis, and drug-induced tubulointerstitial disease. In one patient, the etiology of the patient's renal disease could not be clearly established. All patients were receiving antihypertensive medications, were clinically stable, and were being followed on an outpatient basis. One patient complained of intermittent epistaxis. None of the remaining patients had overt signs of impaired hemostasis.

Each of the eight patients requiring dialysis received hemodialysis three times per week for a total of $12 \mathrm{~h}$ each week. All were clinically stable. Three of these patients received their dialysis at home. Their ages ranged from 48 to $67 \mathrm{yr}$ (mean, $58 \mathrm{yr}$ ), and their serum creatinines 
ranged from 6.9 to $19.1 \mathrm{mg} / \mathrm{dl}$ (mean, $10.8 \mathrm{mg} / \mathrm{dl}$ ). End-stage renal disease was secondary to diabetic nephropathy in two patients; in two patients, it was due to hypertensive renal disease; and in one patient each, it was secondary to membranous nephropathy, polycystic kidney disease, and obstruction plus chronic pyelonephritis. One patient had probable chronic glomerulonephritis, although kidney tissue was not available for evaluation. None of these patients had overt signs of impaired hemostasis.

Of the patients with DIC, four had acute promyelocytic leukemia, two had prostate cancer, and one had pancreatic cancer. Coagulation abnormalities included thrombocytopenia, prolonged prothrombin and thrombin times, increased levels of fibrinogen/fibrin degradation products, and hypofibrinogenemia. Specimen collection and informed consent procedures were approved by the institutions' Human Subjects Review Committees.

Plasma preparation. Venous blood $(9 \mathrm{vol})$, collected by a two-syringe technique, was anticoagulated with a solution (1 vol) of either $3.8 \%$ sodium citrate or citrate containing $90 \mathrm{U} / \mathrm{ml}$ hirudin (Sigma Chemical Co., St. Louis, MO) and 100 kallikrein inhibitor units/ml Trasylol (Mobay Chemical Corp., Pittsburgh, PA). Platelet-poor plasma was separated by centrifugation at $3,000 \mathrm{~g}$ for $15 \mathrm{~min}\left(4^{\circ} \mathrm{C}\right)$. Plasma samples were used immediately or stored at $-70^{\circ} \mathrm{C}$ for subsequent analysis. Pooled normal plasma from 22 individuals was prepared in citrate without added inhibitors.

Assays of Factor VIII/vWF-related activities. VIII:C was measured by one-stage activated partial thromboplastin time (6) using hemophilia A plasma as substrate (VIII:C $<1 \%$ ). $1 \mathrm{U}$ of VIII:C is the amount present in $1 \mathrm{ml}$ of plasma. vWF antigen levels in patient and normal pooled platelet-poor plasma were quantified by Laurell (7) electroimmunoassay, using rabbit anti-human Factor VIII (Calbiochem-Behring Corp., La Jolla, CA) and $0.5 \%$ SeaKem HGT agarose on gel bond backing (Marine Colloids Division, FMC Corp., Rockland, ME).

Previously described procedures were employed to prepare ${ }^{125} \mathrm{I}-\mathrm{Fab}$, quantify VIII:C by immunoradiometric assay (1), and analyze VIII:CAg forms by SDS-4\% PAAGE and densitometry (2).

To determine the ratio of VIII:CAg forms, each sample was analyzed in duplicate at least twice on separate gels. The values presented are the mean of these determinations; standard deviations were within $\pm 10 \%$ of these values. Data were analyzed using the least-squares method of linear regression analysis to calculate Pearson correlation coefficients.

vWF multimer analysis was performed using SDS agarose gels and rabbit ${ }^{125}$ I-labeled anti-human vWF IgG (8). Plasma levels of the prothrombin activation peptide, $F_{1+2}$, were measured by radioimmunoassay $(9,10)$.

Thrombin activation of normal pooled plasma. Normal pooled citrated plasma was incubated for $20 \mathrm{~min}\left(22^{\circ} \mathrm{C}\right)$ with $100 \mathrm{U} / \mathrm{ml}$ Trasylol and 5 mM Gly-Pro-Arg-Pro (Cal Med, South San Francisco, CA). The latter was added to prevent fibrin gel formation after thrombin addition (11). Human thrombin $(2,500 \mathrm{U} / \mathrm{mg}$, sp act; final concentration, $0.05 \mathrm{U} / \mathrm{ml})$, kindly provided by Dr. John Fenton II (New York State Department of Health, Albany, NY), was added at $37^{\circ} \mathrm{C}$ to the plasma solution. At various times, samples were withdrawn for analysis of VIII:C, VIII:CAg concentration, and VIII:CAg molecular weight forms. When VIII:C had dropped back to the initial level, more thrombin (final concentration, $0.05 \mathrm{U} / \mathrm{ml}$ ) was added and the solution was again assayed for Factor VIII-related activities.

\section{Results}

Comparison of VIII:CAg forms in normal controls and in renal dysfunction patients. The distribution of VIII:CAg forms in normal plasma was compared, by ${ }^{125} \mathrm{I}-\mathrm{Fab}-\mathrm{SDS}-\mathrm{PAAGE}$, with that present in patients with renal dysfunction. Normal plasma contained a major $2.4 \times 10^{5} \mathrm{~mol}$ wt VIII:CAg form and a number of minor forms that were relatively quantitatively equivalent. Plasma from renal dysfunction patients, in contrast, had an unusually high percentage of a $1 \times 10^{5} \mathrm{~mol} \mathrm{wt} \mathrm{species} \mathrm{(Fig.} \mathrm{1).} \mathrm{In}$ normal individuals, including men and women from 25 to 65

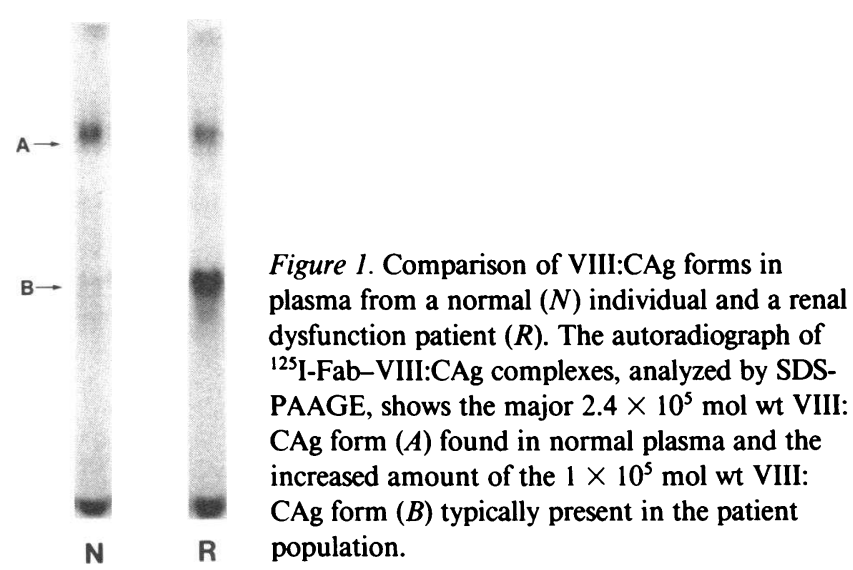

yr old, the mean $2.4 \times 10^{5} / 1 \times 10^{5}$ mol wt VIII:CAg ratio (henceforth referred to as the $2.4 / 1$ VIII:CAg ratio) was $3.3 \pm 0.5$ SD ( $n=22$; range, $2.5-4.6)$. For the dialyzed and undialyzed chronic renal failure patients examined in the present study, this ratio was $1.3 \pm 0.4 \mathrm{SD}(n=19$; range, $0.5-2.1)$.

Correlation of VIII:CAg forms with creatinine, $v W F$, and $F_{1+2}$ concentrations. The increased percentage of the $1 \times 10^{5}$ mol wt VIIl:CAg relative to the $2.4 \times 10^{5} \mathrm{~mol}$ wt form was linearly correlated $(r=0.93, P<0.0001)$ with the log of creatinine concentration (Fig. 2). Patients undergoing dialysis, the group with the highest creatinine values, had the greatest percentage of $1 \times 10^{5} \mathrm{~mol}$ wt VIII:CAg. Undialyzed patients with intermediate creatinine values had 2.4/1 VIII:CAg ratios between those of dialyzed patients and normal individuals.

To be certain that creatinine itself did not cause the increase in the $1 \times 10^{5} \mathrm{~mol}$ wt VIII:CAg form, creatinine (final concentration, $2,5,10,15,20 \mathrm{mg} / \mathrm{dl}$ ) was added to pooled normal plasma. After incubation at $37^{\circ} \mathrm{C}$ for $1 \mathrm{~h}$, the samples were analyzed by ${ }^{125} \mathrm{I}-\mathrm{Fab}-\mathrm{SDS}-\mathrm{PAAGE}$. No increase was observed in the percentage of the $1 \times 10^{5} \mathrm{~mol} \mathrm{wt} \mathrm{VIII:CAg}$. Creatinine levels were also measured in four patients before and after they had undergone dialysis. Despite substantial removal of creatinine, the 2.4/1 VIII:CAg ratio remained abnormal in all cases (Fig. 3).

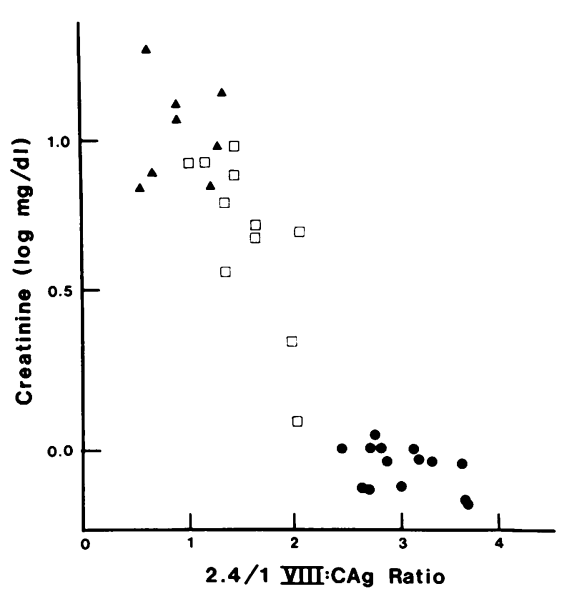

Figure 2. Creatinine concentration $(\log \mathrm{mg} / \mathrm{dl})$ vs. the ratio of 2.4 $\times 10^{5} / 1 \times 10^{5} \mathrm{~mol}$ wt VIII:CAg forms present in normal individuals $(\bullet)$ and undialyzed $(\square)$ and dialyzed (\) renal dysfunction patients. 


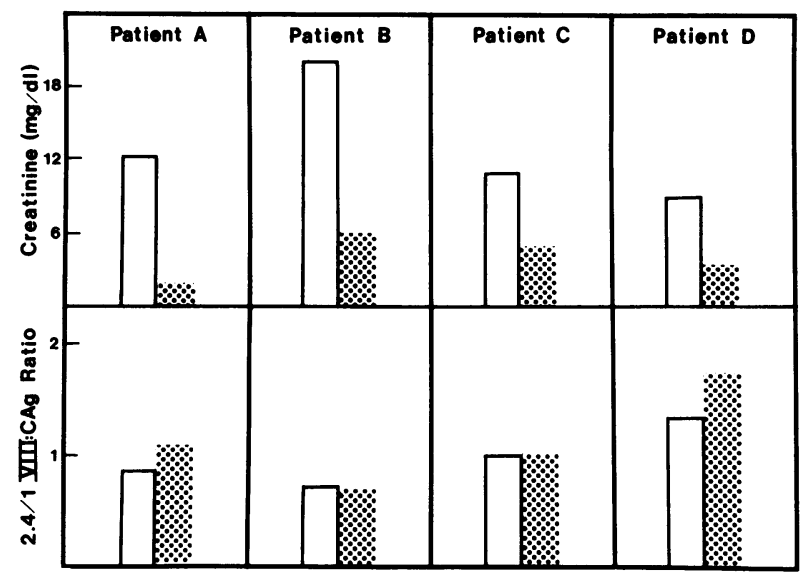

Figure 3. Changes in the creatinine concentration and the $2.4 \times 10^{5} / 1$ $\times 10^{5} \mathrm{~mol}$ wt VIII:CAg ratio in four patients before ( $\square$ ) and after ( $(\mathrm{v})$ dialysis. The normal range of the VIII:CAg ratio is between 2.5 and 4.5.

Since the $1 \times 10^{5} \mathrm{~mol}$ wt VIII:CAg form can be generated in vitro by proteolysis of the $2.4 \times 10^{5} \mathrm{~mol} \mathrm{wt} \mathrm{VIII:CAg} \mathrm{(1),} \mathrm{we}$ considered the possibility that an active enzyme might still be present in the citrated patient plasma. To test this hypothesis, normal citrated plasma ( $9 \mathrm{vol})$ was incubated at $37^{\circ} \mathrm{C}$ for 1,16 , and $20 \mathrm{~h}$ with plasma ( $1 \mathrm{vol})$ from a patient with a $2.4 / 1$ VIII: $\mathrm{CAg}$ ratio of 0.67 . Neither this treatment nor incubation of the patient plasma alone under the same conditions produced an increase in the $1 \times 10^{5} \mathrm{~mol}$ wt VIII:CAg form.

Considering the close association of vWF with VIII:CAg, vWF was also evaluated for evidence of proteolytic degradation. vWF multimers were analyzed using SDS agarose gel electrophoresis, ${ }^{125}$ I-labeled anti-vWF antibody overlay, and autoradiography (8). While vWF levels were generally elevated, all patients, even those with the lowest 2.4/1 VIII:CAg ratios, had normal distributions of $\mathrm{vWF}$ multimers with no evidence of lower molecular weight vWF fragments. Also fibrin-split products were not elevated in any of five patients examined having the lowest 2.4/1 VIII:CAg ratios.

High vWF levels, however, did correlate $(r=0.69$, $P<0.0001)$ with increasing amounts of the $1 \times 10^{5} \mathrm{~mol} \mathrm{wt}$

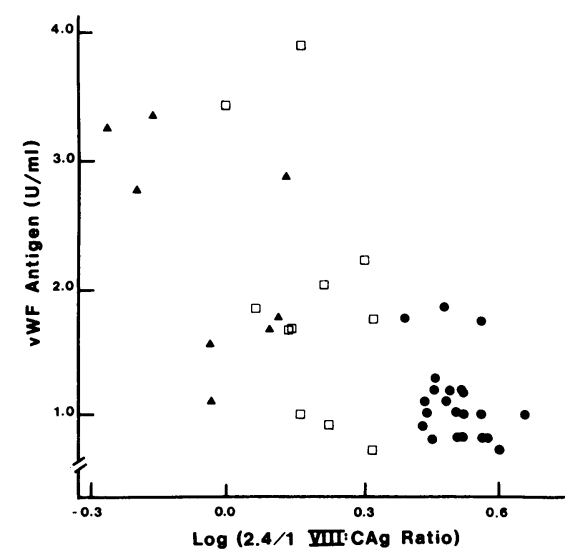

Figure 4. vWF concentration (units per milliliter) vs. the $\log 2.4$ $\times 10^{5} / 1 \times 10^{5} \mathrm{~mol}$ wt VIII:CAg ratio for normal individuals $(\bullet)$ and undialyzed $(\square)$ and dialyzed $(\triangle)$ renal dysfunction patients.
VIII:CAg form, expressed as $\log$ 2.4/1 VIII:CAg ratio (Fig. 4). Those patients with the lowest 2.4/1 VIII:CAg ratios had vWF levels of at least $2.8 \mathrm{U} / \mathrm{ml}$.

In vivo activation of the coagulation system can be determined by measuring the plasma concentration of $F_{1+2}$, a polypeptide cleaved from prothrombin during its transformation to thrombin by Factor Xa. The average value of $F_{1+2}$ for normal individuals is $1.97 \pm 0.97 \mathrm{nM}(\mathrm{SD})(9)$. Renal dysfunction patients generally had high levels of $F_{1+2}$ (Fig. 5). The log of $F_{1+2}$ concentration correlated $(r=0.75, P<0.0001)$ with the patients' abnormal 2.4/1 VIII:CAg ratios.

VIII:CAg forms in patients with DIC. To further analyze the relationship between VIII:CAg forms, $\mathrm{F}_{1+2}$ levels, and renal disease, an additional group of patients $(n=7)$ with DIC but without renal failure was examined. The average $F_{1+2}$ concentration in these patients' plasma, $40.1 \pm 13.7 \mathrm{nmol} /$ liter (range, 21.3-63.4 $\mathrm{nmol} /$ liter), was higher than the average value for renal dysfunction patients of $7.0 \pm 3.7 \mathrm{nmol} /$ liter (range, $2.6-16.2 \mathrm{nmol} /$ liter). Except for one DIC patient with VIII:CAg forms quantitatively comparable to those of normal individuals, DIC patients, like renal dysfunction patients, had a relatively high proportion of their VIII:CAg in the $1 \times 10^{5} \mathrm{~mol} \mathrm{wt}$ form (Fig. 6). Unlike renal dysfunction patients and normal individuals, however, DIC patients generally had a large proportion of VIII:CAg in the $8 \times 10^{4} \mathrm{~mol} \mathrm{wt}$ form.

Changes in Factor VIII-related activities generated by thrombin. In earlier studies $(1,2)$ we showed that in vitro thrombin proteolysis of the $2.4 \times 10^{5} \mathrm{~mol} w \mathrm{wIII}: \mathrm{CAg}$ form yields the $1 \times 10^{5}$ and lower molecular weight VIII:CAg species, a reaction accompanied first by an increase and then a decline in VIII:C. These results support the notion that the $2.4 \times 10^{5} \mathrm{~mol}$ wt VIII:CAg must be proteolytically modified to produce activated VIII:C, analogous to Factor V-Va activation (12). Since patient plasma contained more of the $1 \times 10^{5} \mathrm{~mol} \mathrm{wt} \mathrm{VIII:CAg}$ form than normal, one would also expect the patient's ratio of VIII:C to a unit of VIII:CAg concentration to be different from normal plasma. To determine whether increased levels of the 1 $\times 10^{5} \mathrm{~mol} \mathrm{wt}$ form might be indicative of "activated" or "inactivated" Factor VIII protein, the ratio of VIII:C to VIII:CAg concentration (VIII:C/VIII:CAg) was analyzed with respect to the 2.4/1 VIII:CAg ratio in normal individuals and renal dysfunction patients (Fig. 7). A VIII:C/VIII:CAg ratio above or be-

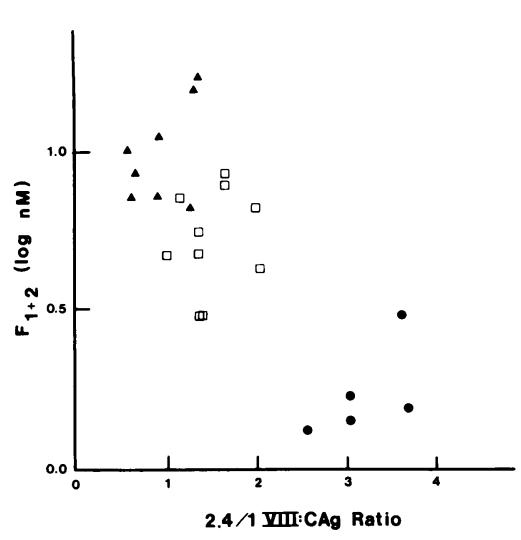

Figure 5. $\mathrm{F}_{1+2}$ concentration $(\log \mathrm{nM})$ vs. the $2.4 \times 10^{5} / 1 \times 10^{5} \mathrm{~mol}$ wt VIII:CAg ratio in samples from normal individuals $(\bullet)$ and undialyzed $(\square)$ and dialyzed $(\triangle)$ renal dysfunction patients. 


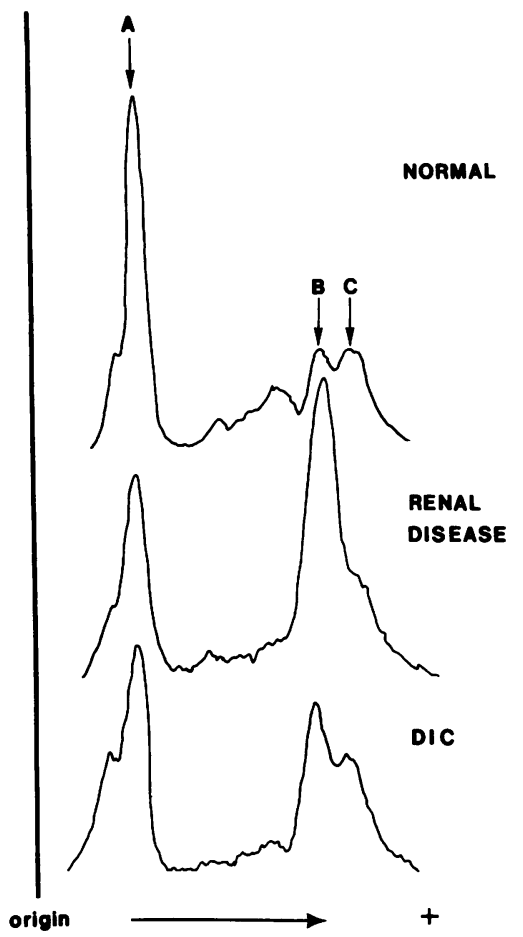

Figure 6. Densitometric scans of autoradiographs obtained by ${ }^{125} \mathrm{I}-$ Fab-SDS-PAAGE analysis of plasma samples from a normal individual, a renal dysfunction patient, and a patient with DIC. In normal individuals, the $2.4 \times 10^{5} \mathrm{~mol} \mathrm{wt}$ VIII:CAg form $(A)$ is predominant, while the $1 \times 10^{5} \mathrm{~mol}$ wt VIII:CAg form $(B)$ is more prominent in the renal disease patient. Both the $1 \times 10^{5}$ and $8 \times 10^{4} \mathrm{~mol}$ wt VIII:CAg forms $(C)$ are increased relative to the $2.4 \times 10^{5} \mathrm{~mol} \mathrm{wt}$ form in patients with DIC.

low the normal range would indicate, respectively, activated or inactivated VIII:C.

Surprisingly, no correlation was found between the activity to antigen ratio and the ratio of VIII:CAg molecular weight forms. With a few high and low exceptions, the patient VIII:C/ VIII:CAg ratio was within the normal range even though the 2.4/1 VIII:CAg ratio was as low as 0.54 in some instances.

To further examine how such a major change in the pro-

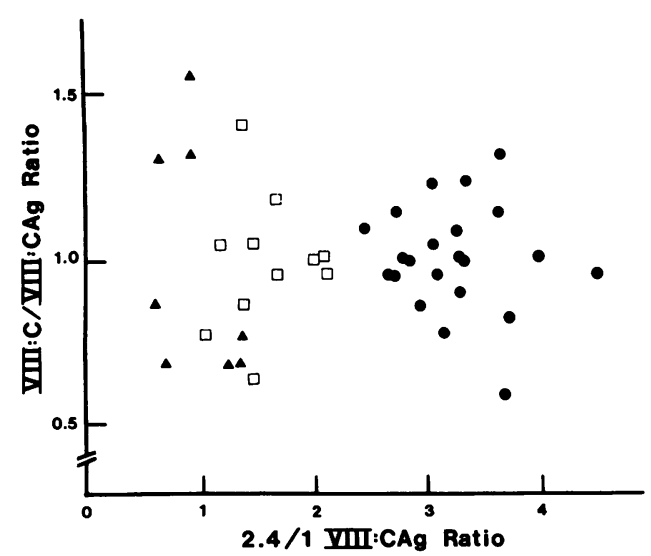

Figure 7. The VIII:C to VIII:CAg ratio vs. the $2.4 \times 10^{5} / 1 \times 10^{5} \mathrm{~mol}$ wt VIII:CAg ratio in samples from normal individuals $(\bullet)$ and undialyzed $(\square)$ and dialyzed $(\Delta)$ renal dysfunction patients. portion of VIII:CAg forms could have so little effect on the VIII: C/VIII:CAg ratio, normal plasma was treated with thrombin and analyzed for variations in VIII:C activity and VIII:CAg forms over time (Fig. 8). Our experimental objective was to see if thrombin proteolysis could generate abnormal VIII:CAg molecular weight ratios but give normal VIII:C-to-VIII:CAg ratios, similar to those present in patient plasma.

Within $3 \mathrm{~min}$ after the addition of thrombin (final concentration, $0.05 \mathrm{U} / \mathrm{ml}$ ) to whole plasma, VIII:C activity rose $12-$ fold and then slowly declined to approximately the initial VIII: $\mathrm{C}$ level $3 \mathrm{~h}$ later. VIII:CAg, measured by a quantitative immunoradiometric assay, changed little over this time period. The 2.4/1 VIII:CAg ratio, in contrast, dropped from 3.7 to $1.81 \mathrm{~min}$ after thrombin addition. The ratio fell to 1.5 at the end of $6 \mathrm{~min}$ and was unchanged at the end of $4 \mathrm{~h}$. In other experiments (not shown) using 0.02 and $0.07 \mathrm{U}$ of thrombin, the 2.4/1 VIII:CAg ratios fell to 2.6 and 0.7 , respectively, and the VIII:C/VIII:CAg ratio returned to the initial value $4 \mathrm{~h}$ after thrombin addition.

More thrombin (final concentration, $0.05 \mathrm{U}$ ) was added to the sample $4 \mathrm{~h}$ after the first addition (Fig. 8). Once again VIII: $C$ rose to a maximum $3 \mathrm{~min}$ after thrombin addition but declined somewhat more rapidly compared with the first activation. The 2.4/1 VIII:CAg ratio decreased from 1.5 to 1 , while the VIII:C/ VIII:CAg ratio returned to the initial level within $2 \mathrm{~h}$. Thrombintreated renal dysfunction plasma gave similar VIII:C activation and inactivation curves (data not shown). Based upon the above data, we infer that the Factor VIII-related properties of renal dysfunction patient plasma in vivo are qualitatively similar to those of a thrombin-treated normal plasma sample, examined at a time when the VIII:C-to-VIII:CAg concentration ratio had declined to a value within the normal range.

\section{Discussion}

Our initial interest in investigating patients with renal dysfunction for potential VIII:C/vWF abnormalities arose from earlier studies (13-15) of the hemostatic mechanism in uremia. Here we have used the ${ }^{125} \mathrm{I}$-Fab-SDS-PAAGE procedure to detect disease-related structural variations of VIII:CAg in this patient group. Additional studies were performed to determine the molecular basis and functional correlates of the unusually high $1 \times 10^{5} \mathrm{~mol}$ wt VIII:CAg concentration found in these patients.

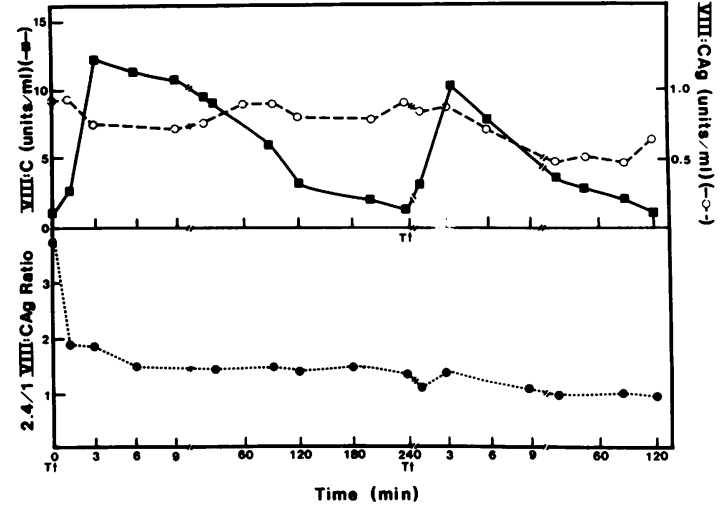

Figure 8. Changes of VIII:C (घ), VIII:CAg (O), and the $2.4 \times 10^{5} / 1$ $\times 10^{5} \mathrm{~mol} \mathrm{wt}$ VIII:CAg ratio $(\bullet)$ in normal pooled plasma treated with thrombin (final concentration, $0.05 \mathrm{U} / \mathrm{ml}$ ) at time zero, followed by a subsequent addition of thrombin (final concentration, $0.05 \mathrm{U} / \mathrm{ml}$ ) at $240 \mathrm{~min}$, when VIII:C had returned to the initial level. 
The severity of renal disease, as evidenced by increasing creatinine levels, was related to the high concentrations of the $1 \times 10^{5} \mathrm{~mol}$ wt VIII:CAg form. The creatinine increase was associated with but did not cause the VIII:CAg abnormality, since removal (Fig. 2) or addition of creatinine had little influence on the $1 \times 10^{5} \mathrm{~mol}$ wt VIII:CAg concentration. Despite the substantial structural modification of VIII:CAg in these patients' plasma, the protein still retained the antigenic and functional activity of unmodified material as analyzed by standard quantitative methods.

We have compared our findings in the above subjects with those in patients with disseminated intravascular coagulation who had markedly increased generation of thrombin within their vascular system as determined by radioimmunoassays for $F_{1+2}$ as well as fibrinopeptide $A$ (10). None of these individuals exhibited evidence of overt renal dysfunction. These patients had not only increased amounts of $1 \times 10^{5} \mathrm{~mol} \mathrm{wt} \mathrm{VIII:CAg}$, but also generally elevated levels of $8 \times 10^{4} \mathrm{~mol} w \mathrm{wIII}: \mathrm{CAg}$.

To examine the molecular basis for the observed VIII:CAg abnormalities in renal dysfunction, we carried out a series of in vitro experiments. Both the VIII:CAg gel pattern and VIII:C-toantigen concentration ratio of renal dysfunction patients could be simulated by treating normal plasma with low concentrations of thrombin. For example, the 2.4/1 VIII:CAg ratios produced by reacting whole plasma with 0.02 and $0.07 \mathrm{U}$ of thrombin (final concentration) bracketed the range of abnormal 2.4/1 VIII: $\mathrm{CAg}$ ratios observed in patient plasmas.

Similarly, the normal ratio of VIII:C to VIII:CAg concentration but abnormal VIII:CAg gel pattern characteristic of renal patients were produced in vitro by incubating normal plasma with $0.05 \mathrm{U}$ of thrombin (Fig. 8). In these experiments, VIII:C rose and fell, but VIII:CAg, measured by a quantitative immunoradiometric assay, remained constant, producing for a time an abnormally high VIII:C/VIII:CAg ratio. Within $4 \mathrm{~h}$, however, the VIII:C/VIII:CAg ratio had returned to initial levels and the VIII:CAg gel pattern was like that of a renal dysfunction patient. Also like the response of a renal dysfunction patient's plasma to thrombin, VIII:C levels rose and fell normally in plasma treated a second time with $0.05 \mathrm{U}$ of thrombin. These results demonstrate that an abnormally high $1 \times 10^{5} \mathrm{~mol}$ wt VIII:CAg level, indicative of proteolysis, is not necessarily accompanied by detectable abnormalities in VIII:C or in the quantitative analysis of VIII:CAg.

Since thrombin-activated VIII:C declined without detectable changes of the $1 \times 10^{5} \mathrm{~mol}$ wt VIII:CAg by the SDS-PAAGE procedure, it is possible that Factor VIII activation and inactivation is not only a function of proteolytic cleavage. A transient enzyme-cofactor complex may form that loses activity through reaction of the enzyme with another plasma protein and/or through a conformational change of the cofactor (16). The 1 $\times 10^{5} \mathrm{~mol}$ wt VIII:CAg may still be available for further complex formation with a thrombinlike enzyme, resulting in apparent VIII:C activation before the complex finally becomes irreversibly degraded. Thrombin proteolysis of VIII:C also produces a number of polypeptides involved in VIII:C activation/inactivation that are not detected by the immunological ${ }^{125}$ I-Fab-SDSPAAGE procedure (17).

With the recent successful cloning of the VIII:C gene (18, 19), it is now known that Factor VIII is synthesized as a single polypeptide chain of $265,000 \mathrm{~mol}$ wt (nonglycosylated). Thrombin, and perhaps other enzymes, partially proteolyze Factor VIII to produce a noncovalently bound complex composed of the protein's 90,000-mol-wt amino terminal and 80,000-mol-wt carboxy terminal ends. This complex may be an activated form of the protein and/or may undergo further proteolysis to produce activated VIII:C. Continued thrombin digestion splits the $90,000-$ mol-wt polypeptide into 50,000- and 43,000-mol-wt fragments, and shortens the 80,000 -mol-wt polypeptide to a 73,000-mol$w t$ form. We are not certain at this point whether ${ }^{125} \mathrm{I}-\mathrm{Fab}$ reacts with the 90,000 - or 80,000 -mol-wt fragment. Thus, while the $2.4 \times 10^{5} \mathrm{~mol} w \mathrm{VIII}: \mathrm{CAg}$ form corresponds to undegraded Factor VIII, the $1 \times 10^{5} \mathrm{~mol} w \mathrm{wI}$ VIII:CAg may be derived from either the amino or carboxy terminal portion of the protein.

Patients with DIC exhibit a different VIII:CAg gel pattern from those with renal dysfunction, consistent with more complete proteolysis by serine proteases such as thrombin. The high levels of both the $8 \times 10^{4}$ and $1 \times 10^{5} \mathrm{~mol} \mathrm{wt} \mathrm{VIII:CAg} \mathrm{forms}$ observed in DIC patients are in accord with prior in vitro studies (2) showing that the $8 \times 10^{4} \mathrm{~mol} \mathrm{wt}$ form is produced after more prolonged exposure of VIII:CAg to thrombin than the $1 \times 10^{5}$ mol wt VIII:CAg form. Our in vivo and in vitro observations therefore support the postulate that a thrombinlike enzyme mediates VIII:CAg degradation in the plasma of patients with either DIC or renal dysfunction.

High $F_{1+2}$ levels were observed in renal dysfunction patients. Without carrying out metabolic clearance studies in this population, it cannot be determined with certainty whether these high levels were the product of an increased rate of in vivo prothrombin activation or a diminished clearance rate of the fragment.

The role that impaired renal and/or reticuloendothelial clearance may play in producing the aberrant $2.4 / 1$ VIII:CAg ratio cannot be assessed from our present data. Milani et al. (20) showed that the degree of reticuloendothelial malfunction in renal disease, measured by the removal of human albumin millimicrospheres, was correlated with elevated vWF levels. In the present study, the abnormally high levels of vWF found in most patients correlated $(r=0.69, P<0.0001)$ with the $\log$ of the 2.4/1 VIII:CAg ratio (Fig. 4), and patients with vWF $>3 \mathrm{U} / \mathrm{ml}$ had low 2.4/1 VIII:CAg ratios. These observations are consistent with the hypothesis that slow removal of degraded VIII:CAg forms is responsible in part for the abnormal 2.4/1 VIII:CAg ratio. However, high vWF levels may also be produced by stimulated or damaged endothelial cells. Although removal of albumin microspheres and high vWF levels might be associated with each other, they could be caused by different mechanisms. Only direct metabolic clearance studies will show whether the abnormal 2.4/1 VIII:CAg ratio is related to prolonged retention of VIII:CAg fragments.

In summary, we have described the first example of a diseaserelated VIII:CAg molecular weight abnormality. Further investigation will be necessary to discover whether this structural modification is unique to the renal patient group and whether this alteration in VIII:C structure correlates with the bleeding diathesis of uremia.

\section{Acknowledgments}

This work was supported in part by National Institutes of Health grants HL-22355, HL28960, and HL33014, and by Veterans Administration Research Funds.

\section{References}

1. Weinstein, M. J., L. E. Chute, and D. Deykin. 1981. Analysis of factor VIII coagulant antigen in normal, thrombin-treated, and hemophilic plasma. Proc. Natl. Acad. Sci. USA. 78:5137-5141. 
2. Weinstein, M. J., and L. E. Chute. 1984. Two distinct forms of Factor VIII coagulant protein in human plasma. Cleavage by thrombin, and differences in coagulant activity and association with von Willebrand factor. J. Clin. Invest. 73:307-316.

3. Weiss, H. J., H. R. Baumgartner, T. B. Tschopp, V. T. Turitto, and D. Cohen. 1978. Correction by factor VIII of the impaired platelet adhesion to subendothelium in von Willebrand's disease. Blood. 51:267279.

4. Rapaport, S. I., S. Schiffman, M. J. Patch, and S. B. Ames. 1963. The importance of activation of antihemophilic globulin and proaccelerin by traces of thrombin in the generation of intrinsic prothrombinase activity. Blood. 21:221-236.

5. Vehar, G. A., and E. W. Davie. 1980. Preparation and properties of bovine factor VIII (antihemophilic factor). Biochemistry. 19:401-410.

6. Langdell, R. D., R. H. Wagner, and K. M. Brinkhous. 1953. Effect of antihemophilic factor on one-stage clotting tests. J. Lab. Clin. Med. 41:637-647.

7. Laurell, C. B. 1972. Electroimmuno assay. Scand. J. Clin. Lab. Invest. 124(Suppl.):21-37.

8. Moake, J. L., J. J. Byrnes, J. H. Troll, C. K. Rudy, M. J. Weinstein, N. M. Colannino, and S. L. Hong. 1984. Abnormal VIII: von Willebrand factor patterns in the plasma of patients with the hemolytic-uremic syndrome. Blood. 64:592-598.

9. Teitel, J. M., K. A. Bauer, H. K. Lau, R. D. Rosenberg. 1982. Studies of the prothrombin activation pathway utilizing radioimmunoassays for the $F_{2} / F_{1+2}$ fragment and thrombin-antithrombin complex. Blood. 59:1086-1097.

10. Bauer, K., and R. D. Rosenberg. 1984. Thrombin generation in acute promyelocytic leukemia. Blood. 64:791-796.

11. Laudano, A., and R. F. Doolittle. 1980. Studies on synthetic peptides that bind to fibrinogen and prevent fibrin polymerization. Structural requirements, number of binding sites, and species differences. Biochemistry. 19:1013-1019.
12. Esmon, C. T. 1979. The subunit structure of thrombin-activated factor V. Isolation of activated factor V, separation of subunits and reconstitution of biological activity. J. Biol. Chem. 254:964-973.

13. Janson, P. A., S. J. Jubelirer, M. J. Weinstein, and D. Deykin. 1980. Treatment of bleeding tendency in uremia with cryoprecipitate. N. Engl. J. Med. 303:1318-1322.

14. Mannucci, P. M., G. Remuzzi, F. Pusineri, R. Lombardi, C. Valsecchi, G. Mecca, and T. S. Zimmerman. 1983. Deamino-8-D-arginine vasopressin shortens the bleeding time in uremia. N. Engl. J. Med. 308: 8-12.

15. Warrell, R. P., M. B. Hultin, and B. S. Coller. 1979. Increased factor VIII/von Willebrand factor activity in renal failure. Am. J. Med. 66:226-228.

16. Hultin, M. B., and J. Jesty. 1981. The activation and inactivation of human factor VIII by thrombin: effect of inhibitors of thrombin. Blood. $57: 476-482$.

17. Weinstein, M. J., C. A. Fulcher, L. E. Chute, and T. S. Zimmerman. 1983. Apparent molecular weight of purified human factor VIII procoagulant protein compared with purified and plasma factor VIII procoagulant protein antigen. Blood. 62:1114-1117.

18. Vehar, G. A., B. Keyt, D. Eaton, H. Rodriguez, D. P. O'Brien, F. Rotblat, H. Oppermann, R. Keck, W. I. Wood, R. N. Harkins, E. G. D. Tuddenham, R. M. Lawn, and D. J. Capon. 1984. Structure of human factor VIII. Nature (Lond.). 312:337-342.

19. Toole, J. J., J. L. Knopf, J. M. Wozney, L. A. Sultzman, J. L. Buecker, D. D. Pittman, R. J. Kaufman, E. Brown, C. Shoemaker, E. C. Orr, G. W. Amphlett, W. B. Foster, M. L. Coe, G. J. Knutson, D. N. Fass, and R. M. Hewick. 1984. Molecular cloning of a cDNA encoding human antihaemophilic factor. Nature (Lond.). 312:342-347.

20. Milani, L., C. Merkel, F. Canel, M. L. Gasparotto, and A. Gatta. 1983. Relationship between plasma levels of factor VIII related antigen and reticulo-endothelial function in chronic uremia. Clin. Nephrol. 20: 235-238. 